\title{
RATIONAL APPROXIMATIONS TO IRRATIONALS
}

\section{ALEXANDER OPPENHEIM}

It is well known that if $p / q$ is a convergent to the irrational number $x$, then $|x-p / q|<1 / q^{2}$. The immediate converse is of course false but I have not seen in the literature ${ }^{1}$ any statement of the converse which is given below.

Theorem 1. If $p$ and $q$ are coprime, $q>0$, and if $|x-p / q|<1 / q^{2}$, then necessarily $p / q$ is one of the three (irreducible) fractions

$$
p^{\prime} / q^{\prime}, \quad\left(p^{\prime}+p^{\prime \prime}\right) /\left(q^{\prime}+q^{\prime \prime}\right), \quad\left(p^{\prime}-p^{\prime \prime}\right) /\left(q^{\prime}-q^{\prime \prime}\right),
$$

where $p^{\prime \prime} / q^{\prime \prime}, p^{\prime} / q^{\prime}$ are two consecutive convergents to the irrational $x$. One at least of the two fractions $\left(p^{\prime}+\epsilon p^{\prime \prime}\right) /\left(q^{\prime}+\epsilon q^{\prime \prime}\right)$ where $\epsilon= \pm 1$ satisfies the inequality.

In other words if the inequality is satisfied, then

$$
p / q=\left[a_{1}, a_{2}, \cdots, a_{n-1}, a_{n}+c\right], \quad c=0, \pm 1,
$$

where $\left[a_{1}, a_{2}, \cdots, a_{r}, \cdots\right]=x$ is the infinite simple continued fraction for $x$, so that the $a_{i}$ are integers, $a_{i} \geqq 1(i \geqq 2)$.

Suppose that $x-p / q=\epsilon \theta / q^{2}, 0<\theta<1, \epsilon= \pm 1$. Let

$$
p / q=\left[b_{1}, b_{2}, \cdots, b_{m}\right], \quad p^{\prime} / q^{\prime}=\left[b_{1}, b_{2}, \cdots, b_{m-1}\right],
$$

where $m$ (which we can choose to be odd or even) is taken so that $(-1)^{m-1}=\epsilon$. Defining $y$ by the equation

$$
x=\left[b_{1}, b_{2}, \cdots, b_{m}, y\right]=\left(y p+p^{\prime}\right) /\left(y q+q^{\prime}\right),
$$

we obtain $\epsilon \theta=q^{2}(x-p / q)=\left(p^{\prime} q-p q^{\prime}\right) q /\left(y q+q^{\prime}\right)$; so that, since $p^{\prime} q-p q^{\prime}=(-1)^{m-1}=\epsilon, y+q^{\prime} / q=1 / \theta$.

Since $1 / \theta>1$ and $q^{\prime} / q<1$ it follows that $y>0$.

If $y>1$, then $y=\left[b_{m+1}, b_{m+2}, \cdots\right]\left(b_{m+1} \geqq 1, \cdots\right)$, and so $x=\left[b_{1}, b_{2}, \cdots, b_{m}, b_{m+1}, \cdots\right]$, which, since the infinite simple continued fraction is unique, shows that $p / q=\left[b_{1}, \cdots, b_{m}\right]$ is the $m$ th convergent to $x$. If however $y<1$, then $1 / y=\left[c, b_{m+1}, b_{m+2}, \cdots\right]$ with $c \geqq 1$. But $q / q^{\prime}=\left[b_{m}, b_{m-1}, \cdots, b_{2}\right]$ and therefore one of $c$ and $b_{m}$ must be unity for, if not, then $1 / y>2, q / q^{\prime}>2, y+q^{\prime} / q<1<1 / \theta$.

${ }^{1}$ Editor's note. In the meantime, R. M. Robinson has proved similar results in the Duke Mathematical Journal, vol. 7 (1940), pp. 354-359. Also the first part of Theorem 1 was observed by P. Fatou, Comptes Rendus de l'Académie des Sciences, Paris, vol. 139 (1904), pp. 1019-1021. 
Hence $x=\left[b_{1}, \cdots, b_{m-1}, b_{m}+c, b_{m+1}, \cdots\right]$ and $b_{i}=a_{i}(i \neq m)$, $b_{m}+c=a_{m}$. Thus $p / q=\left[a_{1}, a_{2}, \cdots, a_{m-1}, b_{m}\right]$ where $b_{m}=1$ or $a_{m}-1$. Consequently

$$
p / q=\left(p_{m-1}+p_{m-2}\right) /\left(q_{m-1}+q_{m-2}\right), \quad b_{m}=1,
$$

or

$$
p / q=\left(p_{m}-p_{m-1}\right) /\left(q_{m}-q_{m-1}\right), \quad b_{m}=a_{m}-1 .
$$

The first part of the theorem is proved.

Now let $p / q=\left(p_{n}+\epsilon p_{n-1}\right) /\left(q_{n}+\epsilon q_{n-1}\right), \epsilon= \pm 1, p_{n} / q_{n}$ being the $n$th convergent to $x=\left[a_{1}, \cdots, a_{n}, x^{\prime}\right]=\left(x^{\prime} p_{n}+p_{n-1}\right) /\left(x^{\prime} q_{n}+q_{n-1}\right)$. Then

$$
q^{2}|x-p / q|=\left|x^{\prime} \epsilon-1\right|\left(q_{n}+\epsilon q_{n-1}\right) /\left(x^{\prime} q_{n}+q_{n-1}\right),
$$

which, since $|\epsilon|=1, x^{\prime}>1$, is less than unity if and only if

$$
\epsilon\left(x^{\prime}-q_{n} / q_{n-1}\right)<2 .
$$

But this inequality is certainly satisfied when $\epsilon$ has the sign opposite to the sign of $x^{\prime}-q_{n} / q_{n-1}$. The second part of the theorem follows.

Irreducible fractions $p / q$ can be divided into three classes $[o / e]$, $[e / o],[o / o]$ in which $o$ and $e$ denote odd and even integers respectively.

Since $p_{n} q_{n-1}-p_{n-1} q_{n}= \pm 1$ it is clear that consecutive convergents $p_{n-1} / q_{n-1}, p_{n} / q_{n}$ belong to two different classes and hence that $\left(p_{n}+\epsilon p_{n-1}\right) /\left(q_{n}+\epsilon q_{n-1}\right)$ where $\epsilon= \pm 1$ must belong to the remaining class of irreducible fractions. It follows from Theorem 1 that for any irrational $x$ infinitely many fractions of each class exist such that $|x-p / q|<1 / q^{2}$.

Theorem 1 in fact determines all such fractions.

This result is due to $\mathrm{Scott}^{2}$ who used the geometric properties of elliptic modular transformations. Scott also showed that the result is the best possible: for a given class and a fixed $k, 0<k<1$, irrationals exist, dense everywhere on the real axis, such that the inequality $|x-p / q|<k / q^{2}$ is satisfied by only a finite number of fractions in the given class.

To prove the last statement it will be enough to show that, if $x=\left[a_{1}, a_{2}, \cdots, a_{n}, \cdots\right]$ where the $a_{n}$ are even integers not less than $2 E+1$, where $E>1$, then for every fraction of type $[o / o]$,

$$
\theta=q^{2}|x-p / q|>1-1 / E .
$$

If $\theta>1$, there is nothing to prove. If $\theta<1$, it follows from our theo-

${ }^{2}$ W. T. Scott, this Bulletin, vol. 46 (1940), pp. 124-129. 
rem that $(p / q$ being irreducible)

$$
p=p_{n}+\epsilon p_{n-1}, \quad q=q_{n}+\epsilon q_{n-1}, \quad \epsilon= \pm 1,
$$

for the convergents to $x$ are all $[e / o]$ or $[o / e]$. Write $X=\left[a_{n+1}\right.$, $\left.a_{n+2}, \cdots\right], Y=\left[a_{n}, a_{n-1}, \cdots, a_{2}\right]$. Then if $n \geqq 2$,

$$
\theta=\frac{(Y+\epsilon)(X-\epsilon)}{X Y+1}=1-\frac{2-\epsilon(X-Y)}{X Y+1}>1-\frac{2+X+Y}{X Y+1},
$$

$$
\begin{aligned}
X Y+1-E(2+X+Y) & =(X-E)(Y-E)-E^{2}-2 E+1 \\
> & (E+1)^{2}-E^{2}-2 E+1>0, \\
& \theta>1-1 / E .
\end{aligned}
$$

If $n=1$, then $p=p_{1}+1, q=q_{1}=1, \theta=1-\left[0, a_{2}, \cdots\right]>1-1 / E$.

Raffles College, Singapore

\section{MEASURABILITY AND DISTRIBUTIVITY IN THE THEORY OF LATTICES ${ }^{1}$}

\section{F. SMILEY}

Introduction. Garrett Birkhoff ${ }^{2}$ derived the following self-dual symmetric condition that a metric lattice be distributive:

$$
\begin{aligned}
2[\mu(a \cup b \cup c)-\mu(a \cap b \cap c)] & =\mu(a \cup b)-\mu(a \cap b)+\mu(a \cup c) \\
& -\mu(a \cap c)+\mu(b \cup c)-\mu(b \cap c) .
\end{aligned}
$$

In a previous note ${ }^{3}$ the author introduced and discussed a generalization of Carathéodory's notion of measurability ${ }^{4}$ with respect to an outer measure function $\mu$ which applies to arbitrary lattices $L$. The $\mu$-measurable elements form a subset $L(\mu)$ consisting of those elements $a \in L$ which satisfy

$$
\mu(a \cup b)+\mu(a \cap b)=\mu(a)+\mu(b)
$$

for every $b \in L$. Closure properties of $L(\mu)$ were investigated. In par-

${ }^{1}$ Presented to the Society, January 1, 1941. The author wishes to express his gratitude to the referee for his valuable suggestions and comments.

${ }^{2}$ Lattice Theory, American Mathematical Society Colloquium Publications, vol. 25 , p. 81 . We shall adopt the notation and terminology of this work and shall indicate specific references to it by $\mathrm{B}$.

${ }^{3} A$ note on measure functions in a lattice, this Bulletin, vol. 46 (1940), pp. 239-241. We shall indicate references to this paper by $\mathrm{M}$.

4 Vorlesungen über Reelle Funktionen, 2d edition, p. 246. 